\title{
A Cognitive Agent Architecture for Feedback Control Scheme Design
}

\author{
Georgios M. Milis, Demetrios G. Eliades, Christos G. Panayiotou, and Marios M. Polycarpou \\ KIOS Research Center for Intelligent Systems and Networks \\ Department of Electrical and Computer Engineering \\ University of Cyprus \\ Nicosia, Cyprus \\ Email: \{milis.georgios, eldemet, christosp, mpolycar\}@ucy.ac.cy
}

\begin{abstract}
We present a novel architecture for the design of feedback control schemes, aiming to automate the cognitive process performed by human experts when designing control schemes for certain systems. The work starts with the identification of types of cyber-physical components participating in a feedback control scheme. Each component is defined as a functional mapping between inputs-vectors and an output vector. The inputs and outputs are then mapped to a semantic space formed by linguistic variables that model the expert knowledge. These semantic annotations of the components are then exploited by a Cognitive Agent with semantic reasoning capabilities, to achieve the online configuration of a feedback control scheme, given a set of specifications and a database of available components' implementations. The adopted knowledge modelling and reasoning techniques are driven by past efforts of the World Wide Web Consortium on the semantic composition of Web services. The applicability of the method is tested via a paper-based enactment of a use-case from the smart buildings domain.
\end{abstract}

\section{INTRODUCTION}

The recent efforts towards the Internet-of-Things (IoT) paradigm, lead to the design of cyber-physical systems [1], which consist of the physical-engineered components and the cyber components that offer advanced internet-enabled communication and computation capabilities. The characteristics of these components turn them promising candidates for the adoption of large-scale systems monitoring and control applications, where the topology and dynamics of the systems are complex and difficult to handle with traditional monolithic architectures. Typically, there is a need for smaller components to work autonomously to accomplish certain tasks in some part of the system and effectively co-operate with the rest of the components so as to address higher-level and heterogeneous monitoring and control challenges [2].

Today's engineered systems more and more employ several cyber and physical components, like sensors for monitoring system states, electrical and mechanical actuators, controllers and a number of other software components for signal processing, estimations and online-learning tasks. Utilising the capabilities of these components in implementing efficient systems' monitoring and control, can considerably help in saving energy, reducing economic cost and improving societal welfare. A great challenged, however, faced in relation with these systems is the fact that their topology and dynamics are subject to changes during their operation. In addition, the components that are utilised for the monitoring and control of their states, may also need to change during operation, due to replacements, availability of new technologies or developments of new monitoring and control capabilities.

During the last two decades, various methodologies have been developed and proposed for system monitoring and control, ranging from applications of the classical control theory of linear systems to techniques with control law adaptation capabilities [3], to methodologies with online learning capabilities of unknown dynamics, which combine model-based analytical redundancy and computational intelligence tools, i.e. neural networks, radial basis functions, etc. [4]. Going one step further, the control community has also been addressing the online re-configurability challenge of control systems [5], including also the identification of newly introduced dynamics in a plug-and-play fusion [6], [7].

Designing a feedback control scheme for a certain system is a complicated procedure which relies on the knowledge and reasoning capabilities of human experts. In practice, a human expert should have a broad background knowledge of tools (e.g., state-of-the-art classical control design methods, non-linear system control methods, online learning methods based on computational intelligence, state-estimation methods, etc.) and in which situations these are best suited, in order to make an informed selection that fully exploits the available measurements, plant and actuation constraints. In practice, it is very rare, if not impossible, to find and employ a human expert of such breadth of knowledge whenever a feedback control scheme is required for a certain system. An additional drawback in current practices is the lack of mechanisms to allow online (and where possible automatic) replacement of individual components or of the overall control scheme.

The above motivate our work for the design of an architecture which is able to utilise expert knowledge and cognitive reasoning based on semantics, to reproduce part of the reasoning procedure of a human expert. This would allow new components to be gradually deployed as they become available, by automatically configuring the feedback control scheme. The aim is to achieve the best composition and to facilitate interoperability with the other available components using a common framework for efficient exchange of data and 
knowledge.

The explicit incorporation of semantics in the control systems' design has been proposed in 1988 by Prof. Ervin Rodin in its article titled "Semantic Control Theory" [8]. Prof. Ervin proposed an architecture, which allows the control system to reason upon the modelled knowledge and select appropriate controllers from a pre-existing pool. A more system-theoretic and philosophical approach to presenting the concepts of semantic control systems can be found in [9]. More recently, concrete examples of using ontological (semantic) knowledge models are observed. For instance in the smart buildings context, DOGont [10] deals with the current issues of domotic environments, that is, the existence of many vendors, each with separate not compatible solutions, the existence of different technologies, different protocols, different device features, etc. Moreover, an early effort introducing the need for a modular architecture for the control system design, has been discussed in [11]. More recent efforts [12], though not focusing specifically on the control problem, address the modelling of cyber-physical systems as collections of services offered by specific cyber-physical tools. In the Computational Intelligence domain, a significant milestone was the approval of the IEEE Standard for Fuzzy Markup Language (IEEE Std 1855-2016), which specifies an interoperability framework for fuzzy logic controllers [13], [14].

The above contributions, however, do not sufficiently address the online evolution and re-configuration of the control systems at component level, which we specifically address in this work. It is emphasized that our work focuses on the online configuration of feedback control schemes, using existing components, and does not focus on the design of any new feedback control algorithm or components.

The envisioned impact from the adoption of a componentbased feedback control design, is the faster exploitation, testing and demonstration of academic research results (such as new learning, control and optimisation algorithms), in industrial applications. To demonstrate the application of the proposed architecture, we present an enactment of a use-case where a Cognitive Agent designs feedback control schemes for smartbuilding-related plants.

The paper is organized as follows: Section II formulates the problem by revisiting the feedback control theory and breaking the feedback control scheme into a set of individual components. Section III then presents the design of the proposed architecture, the knowledge space with the semantic characterization of the components and the Cognitive Agent which utilizes semantic reasoning towards the automatic configuration of feedback control schemes. Section IV presents the use-case enactment. Finally, Section V concludes the paper and discusses future directions.

\section{PROBlem FORMULATION}

Feedback control is defined as the problem of observing the state of a system through available measurements, and utilizing that knowledge together with any additional knowledge about the system's dynamics, to make informed decisions on how to drive available actuators, in order to move or keep the state at a desired trajectory [4]. Typically, a feedback control scheme is specifically designed for a certain system/plant, taking into account the system's measurable variables, known dynamics, available actuation capabilities, as well as other relevant information. The output of the feedback control scheme is the action applied on the system via the system inputs. Considering a discrete-time implementation, at each time $k$, the system input $v(k)$ is a vector of signals that are produced by the actuators affecting the system.

In the general case, the feedback control scheme implementation, can be considered as composed of sub-components, some of which are basic (mandatory) for all implementations of feedback control schemes while others are required only in certain cases. These are discussed in the sequel, adopting the approach introduced in [15] for the component-based analysis of fault-detection schemes.

\section{A. Basic Components}

First of all, a control scheme is always implemented to offer a service on a specific plant, therefore the first basic component of a feedback control scheme is the "Plant" itself. The dynamics of the plant are generally described as

$$
x(k)=f_{p}\left(x(k-1), v(k), w(k), \phi(k), h(k) ; \zeta_{p}\right),
$$

where $x(k) \in \mathcal{R}^{n}$ is the vector of state-variables describing the plant, $f_{p}(\cdot)$ is the function representing the plant's dynamics, $x(k-1)$ is the vector of system-state memory, $v(k) \in \mathcal{R}^{m}$ is the input signal produced by controlled actuators, $w(k)$ is the input signal produced by uncontrolled sources, $\phi(k)$ is a signal modelling faults introduced in the plant's dynamics, $h(k)$ is the input signal produced by third interdependent systems and $\zeta_{p}$ is a set of other plant parameters. (Discrete time is considered for presentation purposes, though the real plant dynamics are typically in continuous time.)

The second basic component of a feedback control scheme is the "Actuator", given by the function:

$$
v(k)=f_{a}\left(u(k) ; \zeta_{a}\right),
$$

where $v(k) \in \mathcal{V}$ is the system input signal discussed earlier, $f_{a}(\cdot)$ is the implementation of a function that produces the signal acting on the controlled system, $u(k)$ is a computed signal that drives the action and $\zeta_{a}$ is a set of other parameters required by the available actuation implementation.

The third basic component in a feedback control scheme is the "Controller" given by the function:

$$
u(k)=f_{c}\left(y(k), r(k), \hat{x}(k), \hat{g}_{p}(k) ; \zeta_{c}\right),
$$

where $u(k)$ is the control decision signal defined in $(2), f_{c}(\cdot)$ is the implementation of a control method to derive the signal, $y(k)$ is the signal representing the plant's feedback as given to the controller, $r(k)$ is the desired system state trajectory, $\hat{x}(k)$ is the estimated system state (optionally used), $\hat{g}_{p}(k)$ is the estimated value of unknown plant dynamics (optionally used) and $\zeta_{c}$ is a set of parameters required by the adopted controller implementation. 
As an example, the control function $f_{c}$ may be a simple bang-bang controller that compares the measured states $y(k)$ with the desired states $r(k)$ and returns a vector of binary signals indicating whether the measurements are greater than the desired values of not. Another example may be a fuzzy control implementation, where the control decision is the defuzzification of a linguistic value, e.g., "fast", which was the output of the triggering of a set of fuzzy rules on the fuzzified system output.

A fourth basic component of a feedback control scheme is the "Sensor", which undertakes the task of measuring the state of the system and is given by the function:

$$
y(k)=f_{s}\left(x(k), v(k), w(k), \phi(k), h(k) ; \zeta_{s}\right),
$$

where $y(k)$ is the signal produced by the installed sensing devices, $f_{s}(\cdot)$ is the implementation of the system measuring given the available sensing devices, $x(k)$ is a vector of the system states, $v(k), w(k), \phi(k), h(k)$ are the various plant's input vectors discussed earlier and $\zeta_{s}$ is a set of parameters required by the adopted implementation of the sensing. For instance, the sensing parameters may correspond to measurement accuracy given by manufacturer or location of the device derived from expert knowledge about the system operation, etc.

In summary, at a minimum, the feedback-control scheme is composed of the functions specified above, forming a composite function $f_{a} \circ f_{c} \circ f_{s}$. That is, the input to the system is a function of the control decision which in turn is a function of the system measurements.

\section{B. Advanced Components}

In addition to the four basic components described in the previous sub-section, additional components may be required by certain feedback-control schemes. For instance, the estimation signal of the system states, $\hat{x}(k)$, may be computed by a separate component. In that case, a "State-Estimation" component can be considered, given by:

$$
\hat{x}(k)=f_{e}\left(\hat{x}(k-1), \hat{g}_{p}(k), y(k), u(k) ; \zeta_{e}\right),
$$

where $\hat{x}(k)$ is the estimated system states signal at the current time step, $f_{e}(\cdot)$ is the adopted implementation of the stateestimation, $y(k)$ and $u(k)$ are the vectors of system's measured output and known control signal respectively, $\zeta_{e}$ is a set of other parameters required by the adopted implementation, $\hat{x}(k-1)$ is the vector of estimated previous system state, and $\hat{g}_{p}(k)$ is the estimated value for unknown plant dynamics (if required). For instance, the State-Estimation component may correspond to a "Kalman filter" which produces estimates based on some prior knowledge about the states, a measurement vector and certain parameters of measurement and state's uncertainty; it can also be a "Luenberger observer" which, based on a known model of system dynamics and the available measurements, produces estimates of the state.

Furthermore, in the case of having a system model with unknown dynamics $g_{p}(\cdot)$ (part of $f_{p}(\cdot)$ ), a "Learning Component" can be utilized, to learn the unknown function using a suitable approximation structure (e.g., neural network, polynomial function, radial-basis functions, wavelets, fuzzy systems, etc), such that $\hat{g_{p}}$ approximates $g_{p}$. This module undertakes the task to learn an unknown part of the overall state dynamics function and can be described in general by:

$$
\hat{g}_{p}(k)=f_{\theta}\left(y(k), u(k) ; \zeta_{\theta}\right)
$$

where $\hat{g}_{p}(k)$ is the estimated value of the unknown function, $f_{\theta}(\cdot)$ is the adopted online learning implementation and $\zeta_{\theta}$ are any other parameters required by the adopted implementation (e.g., the convergence rate, knowledge about the structure of the function). The output of this component may be used as input to components allowing update of the system model on which they base their implementation (e.g., certain StateEstimation and/or Controller components).

In some cases, the measured system output needs to be processed by a separate component before being fed to the controller. For instance, if a system state is measured by more than one sensors, we may want to fuse the measurements and use the computed signal in the controller; alternatively this could correspond to data validation/reconstruction. This step is undertaken by a "Pre-control Function", defined as:

$$
y(k)=f_{y}\left(y_{a}(k) ; \zeta_{y}\right)
$$

where $y(k)$ is now the processed system output, $f_{y}(\cdot)$ is the adopted measurement processing implementation, $y_{a}(k)$ is the actual sensor measurements and $\zeta_{y}$ are any other parameters required by the adopted processing implementation (e.g., knowledge about the proximity of devices to the state location). Then, the controller implementation $f_{c}(\cdot)$, given by (3) receives as input the signal produced by the function $f_{y}(\cdot)$ instead of the actual measurement $y_{a}(k)$.

Similarly, the output of the controller, $u(k)$ may need to be processed before fed to the actuators. For instance, consider the case where a single control signal needs to drive two actuators. This can be implemented by a "Post-control Function", given by:

$$
u_{a}(k)=f_{u}\left(u(k) ; \zeta_{u}\right)
$$

where $u_{a}(k)$ is the processed control decision, $f_{u}(\cdot)$ is the adopted control signal processing implementation, $u(k)$ is the actual control signal and $\zeta_{u}$ are any other parameters required by the adopted processing implementation (e.g., knowledge about the type of actuation devices). Then, the actuators receive the signal $u_{a}(k)$ instead of the control signal $u(k)$.

\section{Components Database}

All implementations of components (functions) of the types discussed above, can be considered as being elements of a function-set $\mathcal{F}$, thus forming a database of components. The set $\mathcal{F}$ is a super-set of the finite-cardinality type-sets of components discussed above, as:

$$
\mathcal{F}=\mathcal{F}_{p} \cup \mathcal{F}_{a} \cup \mathcal{F}_{c} \cup \mathcal{F}_{s} \cup \mathcal{F}_{e} \cup \mathcal{F}_{\theta} \cup \mathcal{F}_{y} \cup \mathcal{F}_{u}
$$




\section{Cognitive Agent}

Depending on the system and the given specifications, an expert engineer would have selected and designed a feedbackcontrol scheme using implementations of all basic components and possibly utilized additional components from the advanced ones, as well as specific domain knowledge expertise. In other words, to make a decision, the expert engineer relies on reasoning which considers the available knowledge about the domain and the feedback-control engineering, including the associated semantics of each component.

The challenge addressed in this work is to design an architecture with the ability to utilize pre-modeled expert knowledge and a set of feedback-control specifications for the online design and configuration of a suitable feedback-control scheme, for a large class of systems. The decision about the configuration of the scheme, can be formulated as:

$$
\sigma=f_{\sigma}(\mathcal{G}, \mathcal{S})
$$

where $\sigma$ is a decision vector that models the selection of specific components from the subsets of $\mathcal{F}$ defined earlier, $f_{\sigma}(\cdot)$ is a function implementing the reasoning and the decision about the scheme configuration, $\mathcal{G}$ is the available experts' knowledge space modelled as a graph, $\mathcal{S}$ is the set of feedback-control specifications given to the function (e.g., the desired state). The elements of $\sigma$ are indexes of the sets of components defined earlier, such that $\sigma=$ $\left[\sigma_{f_{p}}, \sigma_{f_{a}}, \sigma_{f_{c}}, \sigma_{f_{s}}, \sigma_{f_{e}}, \sigma_{f_{\theta}}, \sigma_{f_{y}}, \sigma_{f_{u}}\right]^{\top}$.

Then the configuration of the feedback control scheme can be defined as the operator:

$$
f: \Sigma \times \mathcal{F} \mapsto \mathcal{I}
$$

where $f$ is a generic mapping operator, $\Sigma$ is the space of configuration decisions, $\mathcal{F}$ is the space of all available components (e.g., sensors, actuators, controllers, signal processing functions, etc.), and $\mathcal{I}$ is the space of all configurations of the feedback-control scheme.

The next sections provide details about the proposed architecture for the implementation of the functions $f_{\sigma}$ and $f$, emphasizing on the knowledge space and the reasoning mechanism.

\section{Architecture Design}

The feedback-control architecture, which implements the functions described in the previous section, is depicted in Fig. 1. The top-part of the figure illustrates the Plant on which the feedback-control service is provided. The plant has a controlled input vector $v(k)$, an internal state vector $x(k)$ and dynamics described by a function from the set $\mathcal{F}_{p}$. The plant may be also affected by uncontrolled inputs, i.e. disturbances (vector $w(k)$ ), faults (vector $\phi(k)$ ) and inter-dependencies with other plants (vector $h(k)$ ).

The middle-part of the figure shows the composite Feedback Control Scheme. The input to the feedback-control scheme from the plant, is the vector of measurable properties of the system; we assume these to be the plant's states $x(k)$.

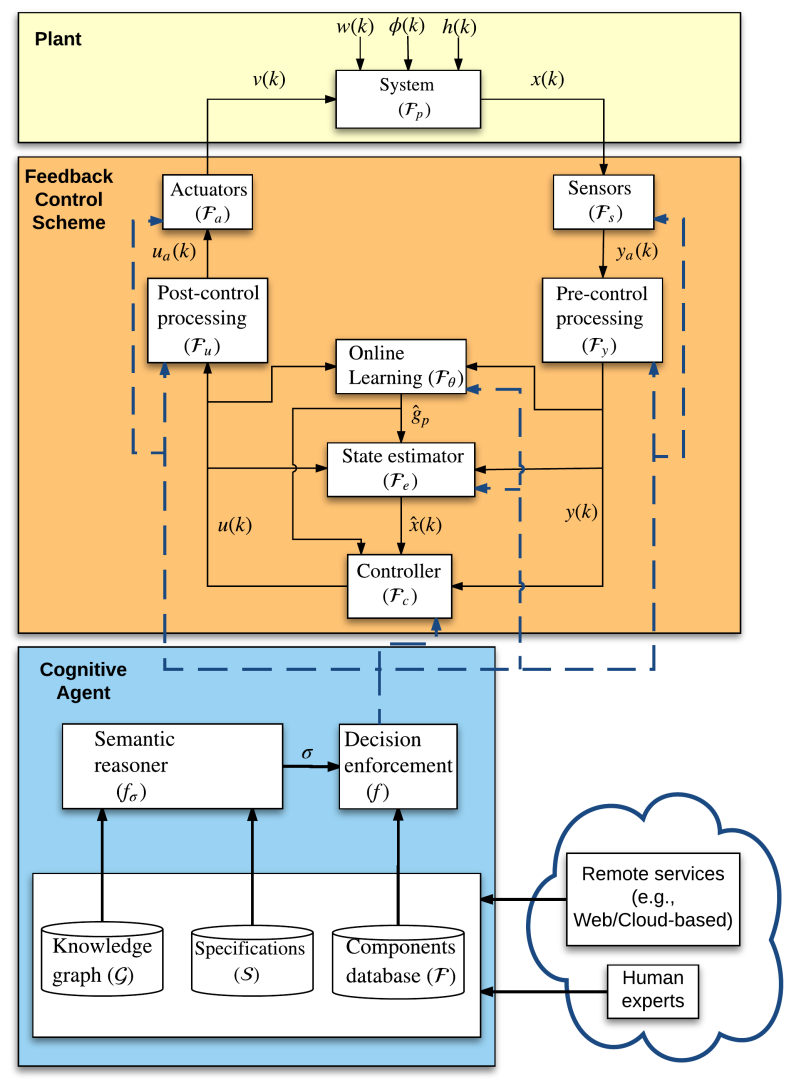

Fig. 1. Block diagram of the architecture. Top: the plant on which feedback control service is provided. Middle: the Feedback Control Scheme. Bottom: the Cognitive Agent

The output is the signal $v(k)$ produced by the actuators and affecting the plant's states. It is noted that the white boxes (functions) refer to the sets of available components of each type, as described in Section II and not to specific implementations. The selection of specific implementations is performed through the decision signal $\sigma$ given by the Cognitive Agent (blue dashed line).

The bottom layer illustrates the design of the Cognitive Agent. As discussed in Section II, the Cognitive Agent function $f_{\sigma}$ utilizes the stored knowledge in the Knowledge Graph $\mathcal{G}$ (including the semantic annotation/characterization of the described components), as well as any given Specifications $\mathcal{S}$, and produces a decision as to what implementations of components to adopt for the feedback control. The decision signal $\sigma$ is passed to the function $f(\cdot)$ that invokes the selected implementations found in the database of components $\mathcal{F}$. An important added-value feature, is that the databases can be also enriched online, via internet-based remote services or directly by human experts, through appropriate interfaces.

The following sub-sections go into details on the implementation of the Cognitive Agent. 


\section{A. Knowledge Space and Cognitive Reasoning}

We have defined all components in a feedback-control scheme as functional mappings between certain inputs to certain outputs. The sets of inputs and outputs of a component are defined here as $\mathcal{T}^{(\omega)}$ and $\mathcal{O}^{(\omega)}$ respectively, where $\omega$ is the component. For instance, the inputs set of a sensor implementation $f_{s}^{1}$ is given by $\mathcal{T}^{\left(f_{s}^{1}\right)}$. The designed architecture considers a fixed choreography of types of components. That is, the plant's output will always be measured by sensing devices, the pre-control processing functions will always use the sensors' output as input and they will give input to a controller, and so on. Therefore, the task of the Cognitive Agent is to check the matchings between outputs and inputs of individual components in consecutive types. This process is called "cognitive reasoning" and will be defined later in a more formal way. Moreover, the matching is performed at a semantic level and is therefore called "semantic matching". In order to check for a semantic matching of an output to an input, the Cognitive Agent first explores the mappings of the output and input variables to an "(expert) knowledge space". These are called "semantic annotations" and are defined as: $\lambda: \mathcal{T}^{(\omega)} \mapsto \Lambda \subset \mathcal{G}$, for inputs and correspondingly for outputs, where $\Lambda$ is the set of all possible semantic annotations of components in the knowledge space.

The semantic matching is defined as another function, $\rho: \Lambda \times \Lambda \mapsto\{$ True, False $\}$. That is, the semantic matching function takes as input a pair of semantic annotations (of an output and an input) and returns "True" if the matching is confirmed and "False" otherwise.

What remains to be defined is the (semantic) knowledge space $\mathcal{G}$ and the actual meaning of the semantic annotation and semantic matching functions. An early version of the knowledge space has been defined, adopting the theory of bipartite graphs, in recent publications by the same authors [16], [17], with case studies from the "Water Distribution Network" and "Smart Grid" domains. In this paper we extent the work adopting a fundamentally similar but formulationwise different approach. We model the expert knowledge with linguistic variables that take values from discrete sets of linguistic terms. For the purposes of this work, the semantic characterization of input and output variables is performed using three properties to which they refer to: "location", "physical quality" and "measurement unit". These correspond to three linguistic variables: $l \in \mathcal{L}$, where $\mathcal{L}$ is the space of possible locations, $q \in \mathcal{Q}$, where $\mathcal{Q}$ is the space of possible physical qualities, and $d \in \mathcal{D}$, where $\mathcal{D}$ is the space of possible measurement units. The combination of these three spaces, defines a 3-dimensional knowledge (discrete) space $(\mathcal{G})$, the elements of which are the semantic annotations of inputs and outputs.

The above is clarified in the sequel through an illustrative example. Consider a plant representing a one-room office with its temperature state. A feedback control scheme is deployed in the office, comprising an electric heater introducing heat energy in the office, a sensor measuring the temperature in degrees Celsius and a simple proportional controller that receives the measurement, compares it to a pre-defined desired value and decides the level on which to operate the heater. In this example we have three components: a sensor $f_{s}^{1}$, an actuator $f_{a}^{1}$ and a controller $f_{c}^{1}$. Also, the plant dynamics are represented by $f_{p}^{1}$. The sensor has an inputs-set $\mathcal{T}^{\left(f_{s}^{1}\right)}$ and an outputs-set $\mathcal{O}^{\left(f_{s}^{1}\right)}$. The respective sets for the other components are defined in the same way. In addition, expert knowledge is assumed defining these linguistic spaces: $\mathcal{L}=\left\{l_{1}\right.$ : 'office', $l_{2}:$ 'ambient', $l_{3}$ : 'ceiling' $\}$, $\mathcal{Q}=\left\{q_{1}:\right.$ 'temperature', $q_{2}$ : 'heat-energy', $q_{3}:$ 'on-off' $\}$ and $\mathcal{D}=\left\{d_{1}:\right.$ 'Celsius', $d_{2}:$ 'Fahrenheit', $d_{3}:$ 'kW', $\left.d_{4}:[1,0]\right\}$. Relations (mappings) between the linguistic terms are also defined. These are formulated with matrix operators, the elements of which take the value 1 if the two terms are semantically related and the value 0 otherwise. For instance, we define the following operators:

$$
\begin{aligned}
& M_{1}: \mathcal{L} \mapsto \mathcal{L}=\begin{array}{ccc}
l_{1} & l_{2} & l_{3} \\
l_{1} \\
l_{2} \\
l_{3}
\end{array}\left[\begin{array}{lll}
1 & 0 & 1 \\
0 & 1 & 0 \\
1 & 0 & 1
\end{array}\right], \\
& M_{2}: \mathcal{L} \mapsto \mathcal{L}=l_{1} \begin{array}{ccc}
l_{1} & l_{2} & l_{3} \\
l_{2} \\
l_{3}
\end{array}\left[\begin{array}{lll}
1 & 1 & 0 \\
1 & 1 & 0 \\
0 & 0 & 1
\end{array}\right],
\end{aligned}
$$

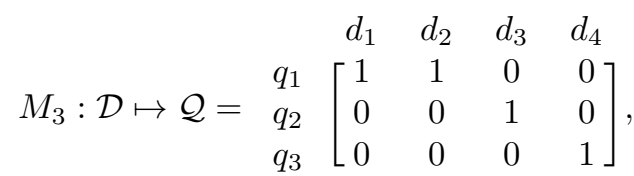

where $M_{1}$ models the relation "is-part-of" or "contains" between two locations, $M_{2}$ models the relation "is-adjacentto" between two locations and $M_{3}$ models the relation "ismeasurement-unit-of" or "is-measured-in-unit" between measurement units and physical qualities.

Given the above described knowledge space, Fig. 2 illustrates the semantic annotation of the input and output of sensor $f_{s}^{1}$, as example. The sensor has one input, $t_{s}$, with semantic annotation $\lambda\left(t_{s}\right)=\left\{l_{3}\right.$ : 'ceiling', $q_{1}$ : 'temperature' $\}$, modelling the fact that the sensor is installed on the ceiling of the room and senses the physical quality "temperature". This is shown on the figure with a green-filled circle mark at the specific point in space. The output of the sensor, $o_{s}$, has a semantic annotation $\lambda\left(o_{s}\right)=\left\{l_{3}\right.$ : 'ceiling', $q_{1}$ : 'temperature', $d_{1}$ : 'Celsius' $\}$, modelling the fact that the sensing signal is further given in degrees Celsius. The output annotation in the knowledge space is shown by a green cross ' + ' mark. For completeness, the defined semantic mappings modelled by the operators $M_{1}, M_{2}, M_{3}$ above, are shown by yellow dashed lines. If two points are connected, it means that if the expert knows something modelled by the first point, the knowledge modelled by the second point can be inferred automatically. Finally, we assume that the desired office temperature has 
been set to $73^{\circ} \mathrm{F}$, which transforms to the following set of (semantic) specifications for the feedback control scheme: $\mathcal{S}=\left\{l_{1}\right.$ : 'office', $q_{1}$ : 'temperature', $d_{2}$ : 'Fahrenheit' $\}$. The specifications are shown on the figure with a green ' $x$ ' mark.

\section{B. Semantic Reasoning Algorithm}

Having defined the above, the reasoning is implemented by the algorithm which is outlined below:

- Start with a set of "Actuator" components that do not violate the specifications.

- Find a "Controller" component with outputs that match semantically with the "Actuators" inputs. If there are remaining inputs and/or required parameters, check whether matching can be achieved by the intervention of "Postcontrol Function" components.

- For the selected controller, find all "Sensor" components that produce outputs that match with the controller's input. Check also "Pre-control Function" components.

- In all cases, check if specifications are met by the selections, otherwise re-iterate within components.

- If all successful, create the decision signal $\sigma$ such as to enforce the selection of the matching components.

This work does not focus on the selection of components based on their performance. Therefore, the Cognitive Agent will implement the feedback control scheme using the first set of components that will be found matching. In case of inability to close the loop with matching components, the algorithm terminates and informs inability to configure a feedback control scheme with currently available components. Moreover, it is noted that the method is currently applied for the configuration of control schemes in plants with slow dynamics, where the time required for the re-configuration of the loop does not affect the stability characteristics. The execution of the algorithm will be clarified through a use-case enactment in the next section.

\section{USE-CASE ENACTMENT}

We consider a use-case where the Cognitive Agent's Semantic Knowledge Space is provided as in Table I. Then, the

TABLE I

Current status of Semantic Knowledge Space

\begin{tabular}{|c|c|}
\hline Specifications & $\begin{array}{l}\mathcal{S}=\left\{l_{1}: \text { 'office', } q_{1}: \text { 'temperature', } d_{2}:\right. \\
\text { 'Fahrenheit' }\}\end{array}$ \\
\hline \multirow[t]{2}{*}{ Plant $f_{p}^{1}$} & Inputs: $\mathcal{T}^{\left(f_{p}^{1}\right)}=\left\{\left\{l_{1}:\right.\right.$ 'office', $q_{2}:$ 'heat-energy' $\left.\}\right\}$ \\
\hline & Outputs: $\mathcal{O}^{\left(f_{p}^{1}\right)}=\left\{\left\{l_{1}:\right.\right.$ 'office', $q_{1}:$ 'temperature' $\left.\}\right\}$ \\
\hline \multirow[t]{2}{*}{ Sensor $f_{s}^{1}$} & Inputs: $\mathcal{T}^{\left(f_{s}^{1}\right)}=\left\{\left\{l_{3}:\right.\right.$ 'ceiling', $q_{1}:$ 'temperature' $\left.\}\right\}$ \\
\hline & $\begin{array}{l}\text { Outputs: } \mathcal{O}^{\left(f_{s}^{1}\right)}=\left\{\left\{l_{3}:\right.\right. \\
\left.\left.\text { 'temperature', 'ceiling', } d_{1}: \text { :Celsius' }\right\}\right\}\end{array}$ \\
\hline \multirow[t]{2}{*}{ Controller $f_{c}^{1}$} & $\begin{array}{l}\text { Inputs: } \mathcal{T}^{\left(f_{c}^{1}\right)}=\left\{\left\{l_{1} \quad: \quad \text { 'office', } q_{1}:\right.\right. \\
\left.\left.\text { 'temperature', } d_{1}: \text { 'Fahrenheit' }\right\}\right\}\end{array}$ \\
\hline & $\begin{array}{l}\text { Outputs: } \mathcal{O}^{\left(f_{c}^{1}\right)}=\left\{\left\{l_{1}: \text { 'office', } q_{3}: \text { 'on-off', } d_{4}\right.\right. \\
[1,0]\}\}\end{array}$ \\
\hline \multirow[t]{2}{*}{ Actuator $f_{a}^{1}$} & $\begin{array}{l}\text { Inputs: } \mathcal{T}^{\left(f_{a}^{1}\right)}=\left\{\left\{l_{3}: \text { 'ceiling', } q_{3}: \text { 'on-off', } d_{4}:\right.\right. \\
[1,0]\}\}\end{array}$ \\
\hline & $\begin{array}{l}\text { Outputs: } \mathcal{O}^{\left(f_{a}^{1}\right)}=\left\{l_{3}: \text { : 'ceiling', } q_{2}:\right. \\
\left.\left.\text { 'heat-energy', } d_{3}: \text { 'kW' }\right\}\right\}\end{array}$ \\
\hline
\end{tabular}

Cognitive Agent starts the execution of its semantic reasoning algorithm to configure the feedback control scheme. The algorithm's steps are illustrated in Fig. 3a. All outputs of components are marked with the cross sign and all their inputs are marked with circles. Moreover, each component is shown with different colour and their inputs and outputs are connected by same colour lines, modelling their internal transfer functions. Finally, the components are numbered in the order they are explored by the algorithm, which helps following the checks for output-input semantic matchings. It can be seen that the semantic annotation of the actuator's output (cross 2) matches the semantic annotation of the plant's input (circle 1) if projected on the "Locations-Physical Property" plane and then move along the semantic relation, which models that the "ceiling" "is-part-of" the "office" (defined earlier by $M_{1}$ operator). Then, the semantic annotation of the controller's output (cross 3) matches the semantic annotation of the actuator's input (circle 2) by considering the relation "contains" defined by the $M_{1}$ operator. Continuing, the semantic annotation of the sensor's output (cross 4) cannot match with the semantic annotation of the controller's input (circle 3), since there is no semantic path to connect them. At this stage, although the rest of the matchings are confirmed, the algorithm terminates stating inability to configure a feedback control scheme with the available components.

However, we consider that the database of components is enriched with a pre-control processing function $f_{y}^{1}$ which converts temperature degrees Celsius to degrees Fahrenheit, as shown in the Table II.

TABLE II

EXTENDING THE KNOWLEDGE SPACE WITH ADDITIONAL KNOWLEDGE

\begin{tabular}{|l|l|}
\hline $\begin{array}{l}\text { Pre-control } \\
\text { proc. } f_{y}^{1}\end{array}$ & Inputs: $\mathcal{T}^{\left(f_{y}^{1}\right)}=\left\{\left\{d_{1}:\right.\right.$ 'Celsius' $\left.\}\right\}$ \\
\hline & Outputs: $\mathcal{O}^{\left(f_{y}^{1}\right)}=\left\{\left\{d_{2}:\right.\right.$ 'Fahrenheit' $\left.\}\right\}$ \\
\hline
\end{tabular}

Fig. $3 b$ shows the semantic annotations of the inputs and outputs of the additional component. It can be seen that the input of the controller (circle 3) can be now connected with the output of the function $f_{y}^{1}$ (cross 4 ) since the latter transforms the signal in relation to the measurement unit. In the same way, the output of the sensor (cross 5) can be connected to the input of the function (circle 4), exploring the semantic relation "is-part-of" between the ceiling and the office. We can also see that the specifications (marked with ' $x$ ') coincide with an output-input semantic matching, which means that the conformance to them is taken into consideration by the system. The algorithm is therefore able to initially configure $(\mathcal{I}=$ 0 ) the feedback control scheme using the set of components $\left\{f_{p}^{1}, f_{a}^{1}, f_{c}^{1}, f_{y}^{1}, f_{s}^{1}\right\}$.

At some future time $k+N$ of the control system operation, the plant becomes equipped with a sensor $\left(f_{s}^{2}\right)$ that measures the opening of a window on the office wall in "percentage". In addition, a new controller $\left(f_{c}^{2}\right)$ is uploaded in the components' database, which implements a fuzzy logic controller based on temperature estimation and window opening inputs, so 


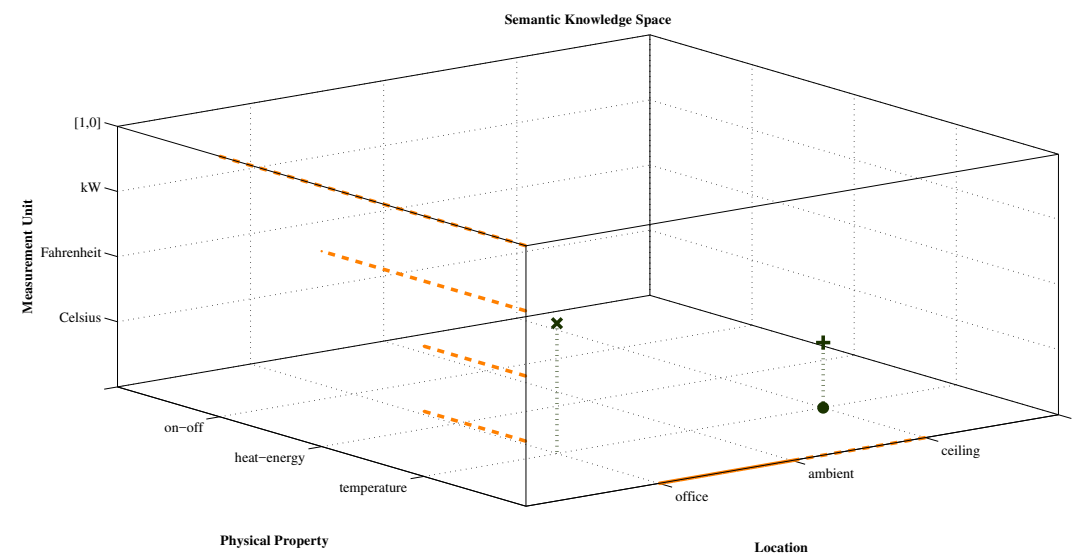

Fig. 2. The current knowledge space, showing the semantic mapping of inputs and outputs of the sensor $f_{s}^{1}$. Input: filled circle mark; Output: Cross ' + ' mark; Semantic mappings: dashed lines; Specifications: ' $\mathrm{x}$ ' mark

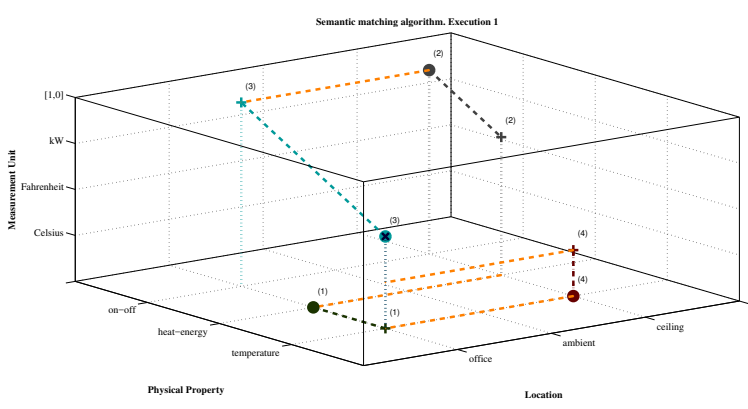

(a)

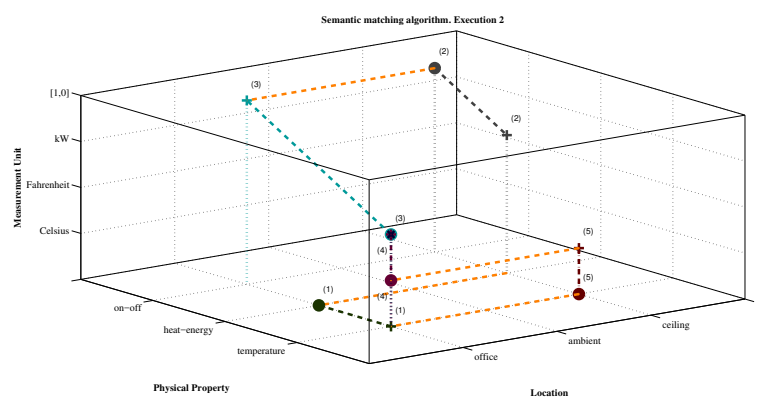

(b)

Fig. 3. Illustration of semantic annotations and matchings

as to make decision for the operation of the heating device. Therefore, a Luenberger observer [18] $f_{e}^{1}$ is also uploaded in the database, able to implement state-estimation for the plant and already considering internally the transformation of the temperature measurement units. The pre-control function that transforms Celsius to Fahrenheit is removed from the database to facilitate clarity of the presentation. The details of the implementations of the components are not the focus of this work, so they are not discussed in detail. Finally, a new specification is introduced, requiring to "consider the opening of the window". The following new semantic annotations are introduced in the knowledge space:

Figure 4 illustrates the execution of the algorithm given the newly introduced changes in the components. It can be seen that nothing changes with the matching of the actuator's output to the plant's input. Then, the algorithm infers that both controllers' outputs are candidates to connect to the input of the actuator (see cross 3,3b). Moreover, the inputs of both controllers (red circles 3,3b) are matched with outputs from available sensors (see light blue cross $5 \mathrm{~b}$ and brown cross 5 through the state estimation component $4 \mathrm{~b}$ ). However, the only option that satisfies also the specification at point $\left\{l_{4}:\right.$ 'window', $q_{4}:$ 'opening' $\}$ is the selection of controller $f_{c}^{2}$
TABLE III

SECOND KNOWLEDGE SPACE EXTENSION

\begin{tabular}{|l|l|}
\hline Sensor $f_{y}^{2}$ & Inputs: $\mathcal{T}^{\left(f_{y}^{2}\right)}=\left\{\left\{l_{4}:\right.\right.$ 'window', $q_{4}:$ 'opening' $\left.\}\right\}$ \\
\hline Controller $f_{c}^{2}$ & $\begin{array}{l}\text { Outputs: } \mathcal{O}^{\left(f_{y}^{2}\right)}=\left\{\left\{l_{4}: \text { 'window', } q_{4}:\right.\right. \\
\left.\left.\text { 'opening', } d_{5}: \text { 'percentage' }\right\}\right\}\end{array}$ \\
\hline $\begin{array}{l}\text { Inputs: } \mathcal{T}^{\left(f_{c}^{2}\right)}=\left\{\left\{l_{1}: \text { 'office', } q_{1}:\right.\right. \\
\left.\text { 'temperature', } d_{2}: \text { 'Fahrenheit' }\right\},\left\{l_{4}:\right.\end{array}$ \\
\hline $\begin{array}{l}\text { Outputs: } \mathcal{O}^{\left(f_{c}^{2}\right)}=\left\{\left\{l_{1}: \text { 'office', } q_{3}: \text { 'on-off', } d_{4}:\right.\right. \\
[1,0]\}\}\end{array}$ \\
\hline$f_{e}^{1}$ & $\begin{array}{l}\text { Inputs: } \mathcal{T}^{\left(f_{e}^{1}\right)}=\left\{\left\{l_{1}: \text { 'office', } q_{1}:\right.\right. \\
\left.\left.\text { 'temperature', } d_{1}: \text { 'Celsius' }\right\}\right\}\end{array}$ \\
\hline Specification $\mathcal{S}$ & $\begin{array}{l}\text { Outputs: } \mathcal{O}^{\left(f_{e}^{1}\right)}=\left\{\left\{l_{1}: \text { 'office', } q_{1}:\right.\right. \\
\left.\left.\text { 'temperature', } d_{2}: \text { 'Fahrenheit' }\right\}\right\}\end{array}$ \\
\hline$\left\{l_{4}:\right.$ 'window', $q_{4}:$ 'opening' $\}$ \\
\hline
\end{tabular}

which uses the measurement of the window opening as well. Therefore, the Cognitive Agent terminates the execution with the successful configuration of the feedback control scheme $(\mathcal{I}=1)$ using the set of components $\left\{f_{p}^{1}, f_{a}^{1}, f_{c}^{2}, f_{e}^{1}, f_{s}^{1}, f_{s}^{2}\right\}$.

In the same way, the Cognitive Agent is able to use more complex knowledge spaces and semantic operations in order to explore the semantic knowledge space and the respective components' semantic annotations and subsequently configure 


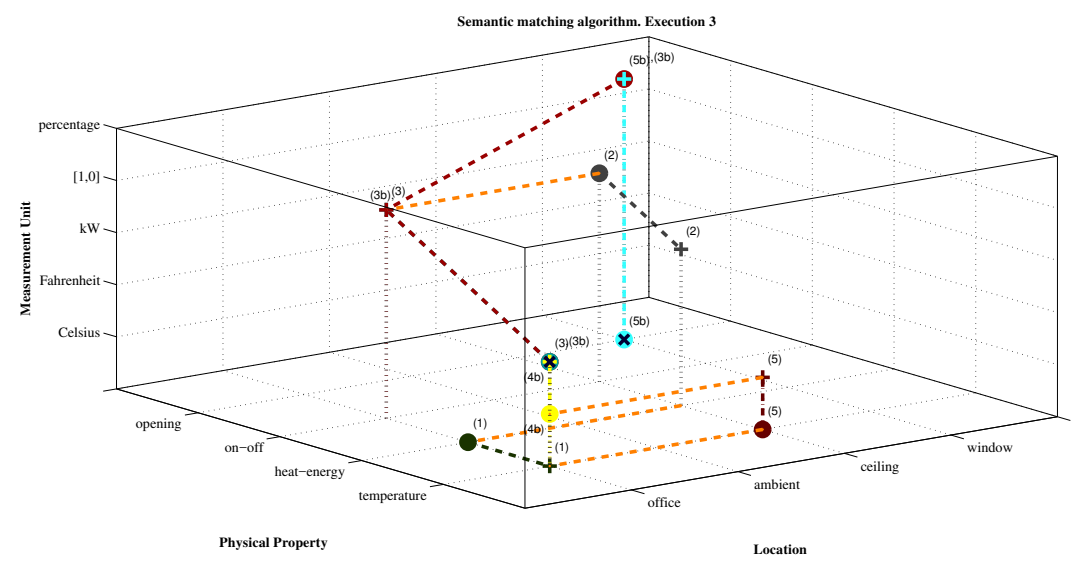

Fig. 4. Illustration of the third semantic matching

the feedback control schemes.

\section{Concluding Remarks and Future Plans}

The work presented in this paper, introduced an effort to automate the design process of a feedback control scheme. The expert engineering and domain knowledge have been modelled using well established knowledge representation techniques and an agent has been designed with the ability to reproducing part of the cognitive process and reasoning performed by the engineer when designing the feedback control scheme. The primary impact of these results is the fact that it defines clear semantic interfaces between parts of the feedback control process and enables industrial set-ups and/or academic prototypes to allow online plugging-in of new implementations of these parts (components) without the need to re-design the whole of the scheme. This is an additional important milestone of our work, while future plans foresee the enrichment of the knowledge graph to facilitate more complex reasoning tasks, the introduction of uncertainty in the semantic mappings and the development of a web platform prototype offering the described services of the Cognitive Agent.

\section{ACKNOWLEDGMENT}

This work is partially funded by the European Research Council (ERC) under the project ERC-AdG-291508 "FaultAdaptive Monitoring and Control of Complex Distributed Dynamical Systems” (FAULT-ADAPTIVE).

\section{REFERENCES}

[1] P. J. Antsaklis, B. Goodwine, V. Gupta, M. McCourt, Y. Po Wu, M. Xia, $\mathrm{H}$. Yu, and Z. Feng, "Control of cyberphysical systems using passivity and dissipativity based methods," Eur. J. Control, vol. 19, no. 5, pp. 379-388, 2013

[2] W. A. H. Thissen and P. M. Herder, "System of Systems Perspectives on Infrastructures," in System of Systems Engineering, M. Jamshidi, Ed. John Wiley \& Sons, Inc., Hoboken, NJ, USA, 2008, ch. 11.

[3] P. A. Ioannou and J. Sun, Robust Adaptive Control. Englewood Cliffs, NJ: Prentice-Hall, 1996.

[4] J. Farrell and M. Polycarpou, Adaptive Approximation Based Control: Unifying Neural, Fuzzy and Traditional Adaptive Approximation Approaches, N. J. W. Hoboken, Ed. J. Wiley, 2006.
[5] M. Blanke, M. Kinnaert, J. Lunze, and M. Staroswiecki, Diagnosis and fault-tolerant control. Springer Verlag, 2003.

[6] T. Knudsen, "Awareness and its use in Plug and Play Process Control," Convergence, pp. 4078-4083, 2009.

[7] J. Stoustrup, "Plug \& Play Control: Control Technology Towards New Challenges," European Journal of Control, vol. 15, no. 3-4, pp. 311-330, Aug. 2009. [Online]. Available: http://ejc.revuesonline.com/ article.jsp?articleId $=13584$

[8] E. Rodin, "Semantic control theory," Appl. Math. Leu., vol. 1, no. 1, 1988.

[9] C. Joslyn, "Semantic control systems," World Futures: Journal of General Evolution, vol. 45, no. 1-4, pp. 87-123, 1995.

[10] E-Lite, "DogOnt," 2012. [Online]. Available: http://elite.polito.it/dogont

[11] M. Boasson, "Control systems software," Automatic Control, IEEE Transactions on, vol. 38, no. 7, pp. 1094-1106, 1993.

[12] J. Huang, F. Bastani, I. L. Yen, J. Dong, W. Zhang, F. J. Wang, and H. J. Hsu, "Extending service model to build an effective service composition framework for cyber-physical systems," in IEEE International Conference on Service-Oriented Computing and Applications, SOCA' 09, 2009.

[13] G. Acampora and V. Loia, "Fuzzy control interoperability and scalability for adaptive domotic framework," IEEE Transactions on Industrial Informatics, vol. 1, no. 2, pp. 97-111, May 2005.

[14] 1855-2016 - IEEE Standard for Fuzzy Markup Language, IEEE Std., 2016.

[15] G. Milis, D. Eliades, C. Panayiotou, and M. Polycarpou, "A cognitive fault-detection design architecture," in IJCNN, World Congress in Computational Intelligence, 2016.

[16] G. M. Milis, D. G. Eliades, C. G. Panayiotou, and M. M. Polycarpou, "Semantic mediation in smart water networks," in Computational Intelligence, 2015 IEEE Symposium Series on, Dec 2015, pp. 617-624.

[17] G. M. Milis, M. Asprou, E. Kyriakides, C. G. Panayiotou, and M. M. Polycarpou, "Semantically-enhanced configurability in state estimation structures of power systems," in Computational Intelligence, 2015 IEEE Symposium Series on, Dec 2015, pp. 679-686.

[18] D. Luenberger, "Observers for multivariable systems," IEEE Transactions on Automatic Control, vol. 11, no. 2, pp. 190-197, Apr 1966.

[19] The Apache Software Foundation, "The Apache Jena project." [Online]. Available: http://jena.apache.org/

[20] D. Martin, M. Burstein, J. Hobbs, O. Lassila, D. McDermott, S. McIlraith, S. Narayanan, M. Paolucci, B. Parsia, T. Payne, E. Sirin, N. Srinivasan, and K. Sycara, "OWL-S: Semantic Markup for Web Services," 2008. [Online]. Available: http://www.ai.sri.com/daml/ services/owl-s/1.2/overview/

[21] A. Sheth, C. Henson, and S. Sahoo, "Semantic Sensor Web," IEEE Internet Computing, vol. 12, no. 4, pp. 78-83, jul 2008. [Online]. Available: http://knoesis.org/library/publications/SHS08-ICColumn-SSW.pdf

[22] W3C, "Semantic Web Query Standards," 2004. [Online]. Available: http://www.w3.org/standards/semanticweb/query 